\title{
Clinical manifestations and pathogen characteristics in children admitted for suspected COVID-19
}

\author{
Xiaofang $\mathrm{Cai}^{1}$, Hanlan Jiang ${ }^{1}$, Simin Zhang ${ }^{1}$, Shengying Xia ${ }^{1}$, Wenhui $\mathrm{Du}^{1}$, Yaoling $\mathrm{Ma}^{1}$, Tao $\mathrm{Yu}^{2}$, Wenbin Li $(\bowtie)^{3}$ \\ ${ }^{I}$ Department of Emergency, Wuhan Children's Hospital (Wuhan Maternal and Child Healthcare Hospital), Tongji Medical College, \\ Huazhong University of Science and Technology, Wuhan 430016, China; ${ }^{2}$ Department of Clinical Laboratory, Wuhan Children's Hospital \\ (Wuhan Maternal and Child Healthcare Hospital), Tongji Medical College, Huazhong University of Science and Technology, Wuhan 430016, \\ China; ${ }^{3}$ Department of Pediatrics, Tongji Hospital, Tongji Medical College, Huazhong University of Science and Technology, Wuhan \\ 430030, China \\ (C) Higher Education Press 2020
}

\begin{abstract}
Coronavirus disease 2019 (COVID-19), which is caused by severe acute respiratory syndrome coronavirus-2 (SARS-CoV-2), has spread around the world. However, approaches to distinguish COVID-19 from pneumonia caused by other pathogens have not yet been reported. We retrospectively analyzed the clinical data of 97 children with probable COVID-19. A total of $13(13.4 \%)$ patients were confirmed positive for SARS-CoV-2 infection by nucleic acid RT-PCR testing, and $41(42.3 \%)$ patients were found to be infected with other pathogens. Notably, no pathogen was detected in $43(44.3 \%)$ patients. Among all patients, $25(25.8 \%)$ had familial cluster exposure history, and $52(53.6 \%)$ had one or more coexisting conditions. Fifteen (15.5\%) patients were admitted or transferred to the PICU. In the 11 confirmed COVID-19 cases, $5(45.5 \%)$ and $7(63.6 \%)$ were positive for IgM and IgG against SARS-CoV-2, respectively. In 22 patients with suspected COVID-19, 1 (4.5\%) was positive for IgG but negative for IgM. The most frequently detected pathogen was Mycoplasma pneumonia (29, 29.9\%). One patient with confirmed COVID-19 died. Our results strongly indicated that the detection of asymptomatic COVID-19 or coexisting conditions must be strengthened in pediatric patients. These cases may be difficult to diagnose as COVID-19 unless etiologic analysis is conducted. A serologic test can be a useful adjunctive diagnostic tool in cases where SARS-CoV-2 infection is highly suspected but the nucleic acid test is negative.
\end{abstract}

Keywords coronavirus disease 2019; pediatrics; emergency; retrospective investigation; severe acute respiratory syndrome coronavirus 2

\section{Introduction}

Coronavirus disease 2019 (COVID-19), which is caused by severe acute respiratory syndrome coronavirus 2 (SARS-CoV-2), has spread around the world, constituting a public health emergency of international concern [1]. SARS-CoV-2 is the seventh coronavirus that has been identified to date that is known to cause human disease. Compared with SARS and Middle East respiratory syndrome (MERS) viruses that were responsible for pandemics in 2003 and 2012, respectively, SARS-CoV-2 is associated with a lower mortality rate but a higher transmission speed [2-5]. Most people, including children, are susceptible to SARS-CoV-2.

Received April 14, 2020; accepted September 4, 2020

Correspondence: Wenbin Li, lwb717299@163.com
However, data on pediatric patients with COVID-19 are lacking. Similar to SARS and MERS, most children infected with COVID-19 have mild clinical presentation unless they have underlying comorbidities or coexisting conditions [6]. As the largest children's hospital in Central China, Wuhan Children's Hospital Affiliated to Tongji Medical College of Huazhong University of Science and Technology, was the first hospital assigned by the Chinese government to treat children with COVID-19 in Hubei Province, China. During the outbreak, only the fever clinic and emergency department remained open to tend to outpatients, all of whom were required to undergo two preexaminations and triage. All febrile or suspected COVID19 cases were referred to the fever clinic, and the others - including critically ill children-were received by the emergency department after pediatric 5-level triage. The majority of children with confirmed COVID-19 were admitted to the hospital through the fever clinic, whereas 
some with probable COVID-19 were admitted from the emergency department. We retrospectively analyzed the clinical data of these children admitted from the emergency department to characterize thoroughly the features of COVID-19 that can be evaluated to distinguish this novel disease from pneumonia caused by other pathogens in pediatric patients. The study was approved by the institutional ethics board of Wuhan Children's Hospital Affiliated to Tongji Medical College of Huazhong University of Science and Technology. Oral consent was obtained from the legal guardians in this study.

\section{Methods}

\section{Participants and COVID-19 diagnostic criteria}

We retrospectively recruited a total of 97 children (excluding newborn cases) with probable COVID-19 who were admitted by the emergency department of Wuhan Children's Hospital from January 30, 2020 to March 15, 2020. The clinical diagnostic criteria for COVID-19 were based on Chinese experts' consensus statement [7].

\section{Data collection}

The patients' medical records were analyzed by the research team at the emergency department of Wuhan Children's Hospital. Epidemiological, clinical, laboratory, and radiological characteristics, as well as treatment and outcome data, were obtained by two trained staff using standardized data collection forms from electronic medical records. After admission, all 97 cases in the study underwent testing for a common set of pathogens, including influenza, parainfluenza virus, respiratory syncytial virus, adenovirus, enterovirus, Mycoplasma, Chlamydia, and Legionella. Routine bacterial and fungal examinations were also performed as necessary.

\section{Real-time RT-PCR assay for SARS-CoV-2}

A confirmed COVID-19 case was defined as positive for SARS-CoV-2 nucleic acid in nasopharyngeal swab and/or anal swabs by real-time RT-PCR according to WHO guidance. Clinical specimens were collected from patients with probable COVID-19. Real-time RT-PCR was performed using a nucleic acid test kit (BGI Biotechnology CO., Ltd., Wuhan, China) according to the manufacturer's instructions.

\section{Serological test for detection of specific IgM and IgG antibodies against SARS-CoV-2}

In the early phase of the COVID-19 pandemic, no suitable assay for SARS-CoV-2 specific IgM and IgG detection was available. Some children with probable COVID-19 underwent serological testing in the hospital starting on March 5, 2020. Serum samples were analyzed for IgM and IgG against SARS-CoV-2 by using chemiluminescence test kits (ShenZhen YHLO Biotech Co., Ltd., Shenzhen, China) following the manufacturer's instructions.

\section{Treatment, release from isolation, and follow-up}

No effective antiviral treatments for COVID-19 have been developed to date $[4,7,8]$. We chose interferon $\alpha-2 b$ aerosol inhalation, ribavirin intravenous infusion, oseltamivir, arbidol, and traditional Chinese medicine oral administration as antiviral therapies for some patients following the recommendations of the Pediatrics Society of Chinese Medical Association [7,9]. Appropriate oxygen support (nasal cannula and noninvasive or mechanical ventilation) was administered to patients according to the severity of hypoxaemia. Glucocorticoid and immunoglobulin were used in severe cases.

If the patient's body temperature (if febrile) returned to normal for more than 3 days, respiratory symptoms remarkably improved, pulmonary images clearly showed that the focus was evidently absorbed, and the nucleic acid test results were negative two consecutive times (the sampling interval was at least 1 day), then the patient was released from isolation or discharged. Constant health monitoring and medical observation for 14 days at the patient's home was recommended. After 2 weeks post discharge, two trained staff followed up the patients via telephone.

\section{Statistical analysis}

Continuous variables were expressed as median and interquartile range (IQR) values and compared with Mann-Whitney U test or Kruskal-Wallis test. Categorical variables were expressed as number (\%) and compared with $\chi^{2}$ test or Fisher's exact test. A two-sided $\alpha$ value $<0.05$ was considered statistically significant. Data were analyzed using SPSS version 20.0 software (SPSS Inc., Chicago, IL, USA), unless otherwise indicated.

\section{Results}

\section{Baseline characteristics and epidemiology}

Owing to the outbreak of COVID-19, Wuhan was put under lockdown starting on January 23, 2020, and nearly all citizens were required to remain at home. Children with probable COVID-19 were admitted to Wuhan Children's Hospital starting on January 30, 2020. The majority of these admissions occurred between January 30 and March 
15. During this period, out of the 4312 outpatients received by the emergency department, $97(2.25 \%)$ with probable COVID-19 were admitted to the hospital. Out of the 97 patients, $13(13.4 \%)$ cases were confirmed (classified as children with confirmed COVID-19), and 41 cases were found to be infected with other pathogens (classified as children with non-COVID-19). However, no pathogen was detected in 43 cases (classified as children with suspected COVID-19) (Fig. 1). At the same time, between January 30 and March 15, a total of 773 children with probable COVID-19 were admitted to the hospital, of which 245 were confirmed as COVID-19 cases, that is, $12.5 \%$ (97/ 773) probable COVID-19 and 5.3\% (13/245) confirmed COVID-19 cases were admitted from the emergency department.

All 97 patients lived in Wuhan within 14 days before the onset of symptoms; 25 (25.8\%) children had documented exposure to one or more adult family members with confirmed or suspected COVID-19. Compared with the 5 $(11.6 \%)$ patients with suspected COVID-19 and 10 (24.4\%) patients with non-COVID-19 causes, the exposure incidence of the 10 (76.9\%) confirmed COVID-19 cases was significantly higher $(P<0.01)$ (Table 1$)$. Out of the 97 patients, $52(53.6 \%)$ had one or more comorbidities or coexisting conditions, including $6(46.2 \%)$ confirmed COVID-19 cases, 27 (62.8\%) suspected COVID-19 cases, and 19 (46.3\%) non-COVID-19 cases. Epilepsy and encephalopathy $(11,11.3 \%)$, acute abdomen (10, $10.3 \%)$, and immunodeficiency $(8,8.2 \%)$ were the most common coexisting conditions (Table 1).

\section{Clinical manifestations}

The median time from symptom onset to hospital admission for the 97 patients was 4.0 days (IQR, 2.0-7.0 days). The median time was shorter in the 13 confirmed COVID-19 cases (2.0 days; IQR, 1.1-4.0 days) than that in the 43 suspected COVID-19 cases (3.0 days; IQR, 2.0-7.0 days) and in the 41 non-COVID-19 cases (4.0 days; IQR, 3.0-7.0 days) $(P=0.047)$. The confirmed COVID-19 cases had a higher incidence of diarrhea $(5,38.5 \%)$ and abdominal pain $(3,23.1 \%)$ than the suspected COVID-

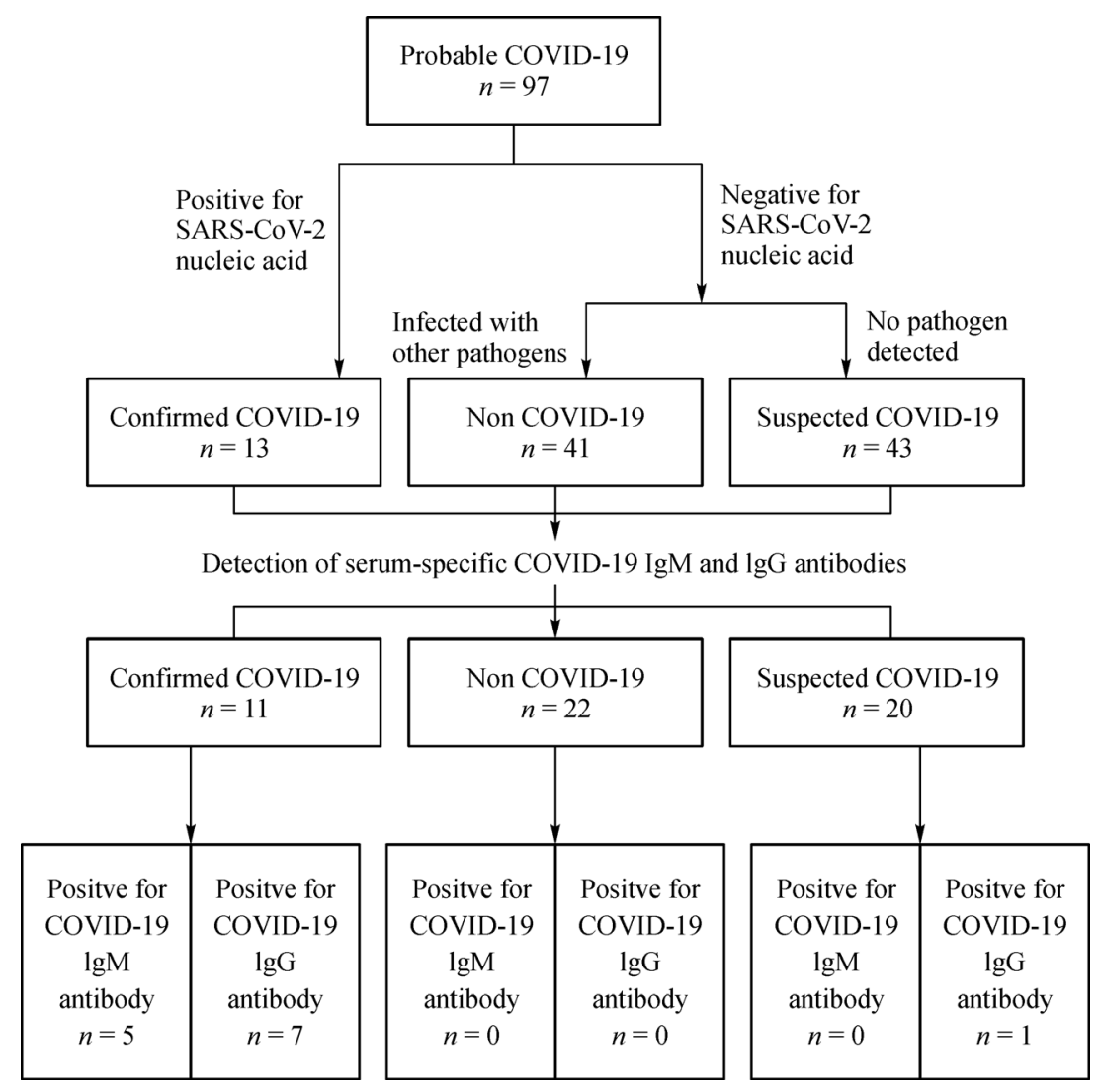

Fig. 1 Detection of COVID-19 nucleic acid and serum-specific IgM and IgG antibodies in 97 probable cases. Of the 97 probable COVID-19 cases, 53 (54.6\%) patients, including 11 (84.6\%) with confirmed COVID-19, 22 (51.2\%) with suspected COVID-19, and 20 (48.8\%) with non-COVID-19, underwent serum anti-SARS-CoV-2 IgM and IgG testing starting on March 5. Out of the 11 confirmed COVID-19 cases, $5(45.5 \%)$ and 7 (63.6\%) were positive for IgM and IgG, respectively. Out of the 22 suspected COVID-19 cases, 1 (4.5\%) was positive for $\operatorname{IgG}$ but negative for $\operatorname{IgM}$. 
Table 1 Baseline characteristics of confirmed COVID-19 cases, suspected COVID-19 cases, and non-COVID-19 cases

\begin{tabular}{|c|c|c|c|c|c|}
\hline & \multicolumn{4}{|c|}{ No. $(\%)$} & \multirow[b]{2}{*}{$P$ value } \\
\hline & Total $(n=97)$ & $\begin{array}{l}\text { Confirmed } \\
\text { COVID-19 case } \\
(n=13)\end{array}$ & $\begin{array}{l}\text { Suspected } \\
\text { COVID-19 case } \\
(n=43)\end{array}$ & $\begin{array}{l}\text { Non-COVID-19 } \\
\text { case }(n=41)\end{array}$ & \\
\hline Age, median (IQR), year & $1.1(0.3-4.5)$ & $2.0(0.8-6.2)$ & $0.6(0.2-1.4)$ & $2.2(0.6-6.5)$ & $0.002 * *$ \\
\hline Sex & & & & & 0.461 \\
\hline Male & $56(57.7)$ & $9(69.2)$ & $26(60.5)$ & $21(51.2)$ & \\
\hline Female & $41(42.3)$ & $4(30.8)$ & $17(39.5)$ & $20(48.8)$ & \\
\hline Family contact history & $25(25.8)$ & $10(76.9)$ & $5(11.6)$ & $10(24.4)$ & $0.000 * *$ \\
\hline $\begin{array}{l}\text { Onset of symptom to hospital admission, } \\
\text { median (IQR), day }\end{array}$ & $4.0(2.0-7.0)$ & $2.0(1.1-4.0)$ & $3.0(2.0-7.0)$ & $4.0(3.0-7.0)$ & $0.047 *$ \\
\hline Comorbidities or coexisting conditions $\mathrm{s}^{\mathrm{a}}$ & $52(53.6)$ & $6(46.2)$ & $27(62.8)$ & $19(46.3)$ & 0.270 \\
\hline Epilepsy and encephalopathy & $11(11.3)$ & 0 & $8(18.6)$ & $3(7.3)$ & \\
\hline Acute abdomen & $10(10.3)$ & $2(15.4)$ & $3(7.0)$ & $5(12.2)$ & \\
\hline Immunodeficiency & $8(8.2)$ & 0 & $6(14.0)$ & $2(4.9)$ & \\
\hline Malignant tumor & $7(7.2)$ & 0 & $5(11.6)$ & $2(4.9)$ & \\
\hline Congenital heart disease & $5(5.2)$ & 0 & $4(9.3)$ & $1(2.4)$ & \\
\hline Trauma & $4(4.1)$ & $2(15.4)$ & $1(2.3)$ & $1(2.4)$ & \\
\hline Connective tissue disease & $3(3.1)$ & 0 & $2(4.7)$ & $1(2.4)$ & \\
\hline Others & $7(7.2)$ & $2(15.4)$ & $1(2.3)$ & $4(9.8)$ & \\
\hline \multicolumn{6}{|l|}{ Symptoms during the course of the disease } \\
\hline Fever & $57(58.8)$ & $7(53.8)$ & $19(44.2)$ & $31(75.6)$ & $0.013^{*}$ \\
\hline Cough & $39(40.2)$ & $3(23.1)$ & $18(41.9)$ & $18(43.9)$ & 0.393 \\
\hline Shortness of breath & $19(19.6)$ & $4(30.8)$ & $6(14.0)$ & $9(22.0)$ & 0.360 \\
\hline Vomiting & $15(15.5)$ & $4(30.8)$ & $4(9.3)$ & $7(17.1)$ & 0.160 \\
\hline Diarrhea & $13(13.4)$ & $5(38.5)$ & $7(16.3)$ & $1(2.4)$ & $0.013 *$ \\
\hline Abdominal pain & $7(7.2)$ & $3(23.1)$ & $1(2.3)$ & $3(7.3)$ & $0.040^{*}$ \\
\hline Fatigue & $10(10.1)$ & $2(15.4)$ & $4(8.9)$ & $4(9.8)$ & 0.787 \\
\hline PICU admission & $15(15.2)$ & $3(23.1)$ & $4(8.9)$ & $8(19.5)$ & 0.271 \\
\hline Length of hospital stay, median (IQR), day & $\begin{array}{l}10.0(8.0-15.0) \\
(n=97)\end{array}$ & $\begin{array}{l}15.0(11.0-16.5) \\
(n=13)\end{array}$ & $\begin{array}{l}10.0(8.0-14.0) \\
(n=43)\end{array}$ & $\begin{array}{l}9.0(8.0-14.5) \\
(n=41)\end{array}$ & 0.080 \\
\hline
\end{tabular}

${ }^{*} P<0.05,{ }^{* * P}<0.01$. ${ }^{\mathrm{a}}$ Out of the 43 suspected COVID-19 cases, 4 had two comorbidities or coexisting conditions and 2 had three comorbidities or coexisting diseases.

Abbreviations: IQR, interquartile range; PICU, pediatric intensive care unit.

19 cases $(7(16.3 \%)$ and $1(2.3 \%)$, respectively) and the non-COVID-19 cases $(1(2.4 \%)$ and $3(7.3 \%)$, respectively) ( $P=0.013$ and 0.040 , respectively). The incidence of fever was higher in non-COVID-19 cases $(31,75.6 \%)$ than that in confirmed COVID-19 cases $(7,53.8 \%)$ and suspected COVID-19 cases $(19,44.2 \%)(P=0.013)$. A total of 19 patients with shortness of breath received oxygen, of which 4 (30.8\%) were confirmed COVID-19 cases, $6(14.0 \%)$ were suspected COVID-19 cases, and 9 $(22.0 \%)$ were non-COVID-19 cases. Out of the 97 patients, $15(15.2 \%)$ were admitted or transferred to pediatric intensive care unit because of organ dysfunction (Table 1).

\section{Laboratory findings and chest radiograph}

White blood cell (WBC) count was normal in 59 (60.8\%) out of the 97 patients; 4 (30.8\%) of the 13 confirmed
COVID-19 cases, 6 (14.0\%) of the 43 suspected COVID19 cases, and $7(17.1 \%)$ of the 41 non-COVID-19 cases showed leucopenia (WBC count $<5.5 \times 10^{9} / \mathrm{L}$ ). Most patients had slightly elevated C-reactive protein $(5.00 \mathrm{mg} / \mathrm{L}$; IQR, $0.75-23.00 \mathrm{mg} / \mathrm{L})(54$ out of 97 cases $(55.7 \%))$ and procalcitonin $(0.12 \mathrm{ng} / \mathrm{mL}$; IQR, $0.07-$ $0.42 \mathrm{ng} / \mathrm{mL}$ ) (69 out of 84 cases $(82.1 \%)$ ) (Table 2).

Out of the 97 patients, alanine aminotransferase and aspartate aminotransferase were elevated in $12(12.4 \%)$ and $35(36.1 \%)$ cases, respectively. Out of 91 patients, increased lactate dehydrogenase, creatine kinase (CK), and CK isoenzyme levels were observed in 23 (25.3\%), 18 (19.8\%), and $62(68.1 \%)$ cases, respectively. Out of 95 patients, blood urea nitrogen and creatinine were elevated in $7(7.4 \%)$ and $3(3.2 \%)$ cases, respectively (Table 2$)$.

Out of 42 patients, plasma concentrations of interleukin (IL)-6 and IL-10 increased in $16(38.1 \%)$ and 20 (47.6\%) cases (mostly severe), respectively. However, IL-2, IL-4, 
Table 2 Laboratory findings of confirmed COVID-19 cases, suspected COVID-19 cases, and non-COVID-19 cases

\begin{tabular}{|c|c|c|c|c|c|c|}
\hline & \multirow[b]{2}{*}{ Normal range } & \multicolumn{4}{|c|}{ Median (IQR) or No. (\%) } & \multirow[b]{2}{*}{$P$ value } \\
\hline & & Total $(n=97)$ & $\begin{array}{l}\text { Confirmed COVID-19 } \\
\text { case }(n=13)\end{array}$ & $\begin{array}{l}\text { Suspected COVID-19 } \\
\text { case }(n=43)\end{array}$ & $\begin{array}{l}\text { Non-COVID-19 } \\
\text { case }(n=41)\end{array}$ & \\
\hline $\begin{array}{l}\text { White blood cell count, } \\
\times 10^{9} / \mathrm{L}\end{array}$ & $5.50-12.00$ & $7.66(6.08-10.98)$ & $6.99(4.60-10.15)$ & $8.49(6.69-12.53)$ & $7.66(6.08-11.02)$ & 0.477 \\
\hline$<5.5$ & & $17(17.5)$ & $4(30.8)$ & $6(14.0)$ & $7(17.1)$ & 0.375 \\
\hline $\begin{array}{l}\text { Neutrophil count, } \\
\times 10^{9} / \mathrm{L}\end{array}$ & $1.08-5.90$ & $3.10(1.64-4.93)$ & $2.26(1.72-5.45)$ & $3.04(1.57-4.94)$ & $3.78(2.03-6.48)$ & 0.143 \\
\hline $\begin{array}{l}\text { Lymphocyte count, } \\
\times 10^{9} / \mathrm{L}\end{array}$ & $1.15-6.00$ & $3.31(2.01-5.70)$ & $2.96(2.25-3.88)$ & $4.66(2.82-6.01)$ & $2.93(1.71-5.29)$ & 0.117 \\
\hline$<1.15$ & & $8(8.2)$ & $1(7.7)$ & $4(9.3)$ & $3(7.3)$ & 0.944 \\
\hline $\begin{array}{l}\text { Platelet count, } \\
\times 10^{9} / \mathrm{L}\end{array}$ & $100-378$ & $324(231-421)$ & $314(219-358)$ & $376(238-541)$ & $306(213-368)$ & 0.077 \\
\hline$<100$ & & 4 & $1(7.7)$ & $2(4.7)$ & $1(2.4)$ & 0.690 \\
\hline $\mathrm{CRP}, \mathrm{mg} / \mathrm{L}$ & $0-3.00$ & $5.00(0.75-23.00)$ & $5.01(1.02-27.55)$ & $2.88(0.75-15.30)$ & $10.00(0.75-35.40)$ & 0.171 \\
\hline PCT, ng/mL & $\leqslant 0.05$ & $\begin{array}{l}0.12(0.07-0.42) \\
(n=84)\end{array}$ & $\begin{array}{l}0.07(0.04-0.38) \\
(n=12)\end{array}$ & $\begin{array}{l}0.11(0.07-0.34) \\
(n=34)\end{array}$ & $\begin{array}{l}0.16(0.09-0.66) \\
(n=38)\end{array}$ & 0.215 \\
\hline ALT, U/L & $9-52$ & $23(15-36.5)$ & $21(14.5-62)$ & $28(22-38)$ & $19(13-28)$ & $0.024 *$ \\
\hline $\mathrm{AST}, \mathrm{U} / \mathrm{L}$ & $15-46$ & $39(28-57)$ & $46(25-104)$ & $41(32-57)$ & $33(26-56)$ & 0.239 \\
\hline $\mathrm{LDH}, \mathrm{U} / \mathrm{L}$ & $161-371$ & $\begin{array}{l}284(223-372) \\
(n=91)\end{array}$ & $\begin{array}{l}344(237-656.5) \\
(n=13)\end{array}$ & $\begin{array}{l}285(231-387) \\
(n=38)\end{array}$ & $\begin{array}{l}270(214-352) \\
(n=40)\end{array}$ & 0.224 \\
\hline $\mathrm{CK}, \mathrm{U} / \mathrm{L}$ & $30-170$ & $\begin{array}{l}89(69-161) \\
(n=91)\end{array}$ & $\begin{array}{l}152(90.5-248.5) \\
(n=13)\end{array}$ & $\begin{array}{c}88(68-165) \\
(n=38)\end{array}$ & $\begin{array}{c}86(64-141) \\
(n=40)\end{array}$ & $0.042 *$ \\
\hline CK isoenzyme, $\mathrm{U} / \mathrm{L}$ & $0-24$ & $\begin{array}{c}34(22-47 \\
(n=91)\end{array}$ & $\begin{array}{l}34(19.5-94) \\
(n=13)\end{array}$ & $\begin{array}{l}36(27-49) \\
(n=38)\end{array}$ & $\begin{array}{c}26(19-43) \\
(n=40)\end{array}$ & 0.152 \\
\hline $\mathrm{BUN}, \mu \mathrm{mol} / \mathrm{L}$ & $2.5-6.1$ & $\begin{array}{l}3.1(2.3-4.2) \\
(n=95)\end{array}$ & $\begin{array}{l}3.5(2.3-5.6) \\
(n=12)\end{array}$ & $\begin{array}{c}2.5(2.0-4.3) \\
(n=43)\end{array}$ & $\begin{array}{c}3.3(2.8-3.8) \\
(n=40)\end{array}$ & 0.118 \\
\hline $\mathrm{Cr}, \mu \mathrm{mol} / \mathrm{L}$ & $46-92$ & $\begin{array}{c}24(21-30) \\
(n=95)\end{array}$ & $\begin{array}{c}26(24-61) \\
(n=12)\end{array}$ & $\begin{array}{c}23(20-28) \\
(n=43)\end{array}$ & $\begin{array}{c}26(21-37) \\
(n=40)\end{array}$ & 0.162 \\
\hline PT, s & $10.2-13.4$ & $\begin{array}{l}11.6(11.0-12.4) \\
(n=57)\end{array}$ & $\begin{array}{l}11.4(10.9-12.2) \\
(n=10)\end{array}$ & $\begin{array}{l}11.6(10.9-12.3) \\
(n=21)\end{array}$ & $\begin{array}{l}11.8(11.1-12.7) \\
(n=26)\end{array}$ & 0.567 \\
\hline APTT, s & $25.7-39$ & $\begin{array}{l}31.0(27.6-35.8) \\
(n=57)\end{array}$ & $\begin{array}{l}31.4(30.0-39.2) \\
(n=10)\end{array}$ & $\begin{array}{l}31.3(26.9-37.7) \\
(n=21)\end{array}$ & $\begin{array}{l}30.0(27.6-34.3) \\
(n=26)\end{array}$ & 0.609 \\
\hline D-dimer & $0-0.55$ & $\begin{array}{l}0.45(0.31-1.49) \\
(n=25)\end{array}$ & $\begin{array}{l}0.92(0.40-12.35) \\
(n=6)\end{array}$ & $\begin{array}{l}0.45(0.28-2.31) \\
\quad(n=9)\end{array}$ & $\begin{array}{l}0.41(0.28-0.56) \\
(n=10)\end{array}$ & 0.326 \\
\hline \multicolumn{7}{|l|}{ Cytokine, $\mathrm{pg} / \mathrm{mL}$} \\
\hline IL-2 & $0-11.4$ & $\begin{array}{c}1.6(1.3-1.8) \\
(n=42)\end{array}$ & $\begin{array}{l}1.4(1.3-1.8) \\
(n=7)\end{array}$ & $\begin{array}{l}1.7(1.3-2.2) \\
(n=15)\end{array}$ & $\begin{array}{c}1.5(1.3-1.7) \\
(n=20)\end{array}$ & 0.408 \\
\hline IL-4 & $0-12.9$ & $\begin{array}{l}2.5(2.1-3.4) \\
(n=42)\end{array}$ & $\begin{array}{l}3.01(2.30-3.78) \\
\quad(n=7)\end{array}$ & $\begin{array}{l}2.2(1.9-4.0) \\
(n=15)\end{array}$ & $\begin{array}{l}2.4(2.1-2.8) \\
(n=20)\end{array}$ & 0.572 \\
\hline IL-6 & $0-20.9$ & $\begin{array}{l}9.3(4.8-30.5) \\
(n=42)\end{array}$ & $\begin{array}{l}70.83(7.06-177.86) \\
(n=7)\end{array}$ & $\begin{array}{l}6.6(4.7-27.5) \\
(n=15)\end{array}$ & $\begin{array}{l}11.4(4.3-28.1) \\
(n=20)\end{array}$ & 0.323 \\
\hline IL-10 & $0-5.9$ & $\begin{array}{l}5.9(4.4-8.7) \\
(n=42)\end{array}$ & $\begin{array}{l}6.68(3.31-26.85) \\
(n=7)\end{array}$ & $\begin{array}{c}5.9(4.4-9.5) \\
(n=15)\end{array}$ & $\begin{array}{c}5.7(4.7-7.9) \\
(n=20)\end{array}$ & 0.968 \\
\hline TNF- $\alpha$ & $0-5.5$ & $\begin{array}{c}1.8(1.3-2.4) \\
(n=42)\end{array}$ & $\begin{array}{l}2.1(1.1-3.5) \\
(n=7)\end{array}$ & $\begin{array}{l}2.2(1.6-2.6) \\
(n=15)\end{array}$ & $\begin{array}{c}1.6(1.2-2.1) \\
(n=20)\end{array}$ & 0.200 \\
\hline \multicolumn{7}{|l|}{$\begin{array}{l}\text { TBNK lymphocyte } \\
\text { detection, } \mu \mathrm{L}^{-1}\end{array}$} \\
\hline $\mathrm{CD}^{+} \mathrm{T}$ & $805-4459$ & $\begin{array}{l}2056(1188-3098) \\
(n=35)\end{array}$ & $\begin{array}{l}2175(1060-4014) \\
(n=9)\end{array}$ & $\begin{array}{l}1860(1065-3477) \\
(n=10)\end{array}$ & $\begin{array}{l}2196(890-2666) \\
(n=16)\end{array}$ & 0.949 \\
\hline $\mathrm{CD} 8^{+} \mathrm{T}$ & $314-2080$ & $\begin{array}{l}766(258-1120) \\
(n=35)\end{array}$ & $\begin{array}{l}1068(539-1335) \\
(n=9)\end{array}$ & $\begin{array}{l}770(152-977) \\
(n=10)\end{array}$ & $\begin{array}{l}538(251-1012) \\
(n=16)\end{array}$ & 0.336 \\
\hline $\mathrm{CD}^{+}{ }^{+} \mathrm{T}$ & $345-2350$ & $\begin{array}{l}1149(549-2168) \\
(n=35)\end{array}$ & $\begin{array}{l}1070(446-2921) \\
(n=9)\end{array}$ & $\begin{array}{l}1054(763-2332) \\
(n=10)\end{array}$ & $\begin{array}{l}1240(498-1725) \\
(n=16)\end{array}$ & 0.981 \\
\hline $\mathrm{CD} 16^{+} \mathrm{CD} 56^{+} \mathrm{NK}$ & $210-1514$ & $\begin{array}{l}276(139-516) \\
(n=35)\end{array}$ & $\begin{array}{l}311(102-516) \\
(n=9)\end{array}$ & $\begin{array}{l}289(147-555) \\
(n=10)\end{array}$ & $\begin{array}{l}245(125-533) \\
(n=16)\end{array}$ & 0.939 \\
\hline
\end{tabular}




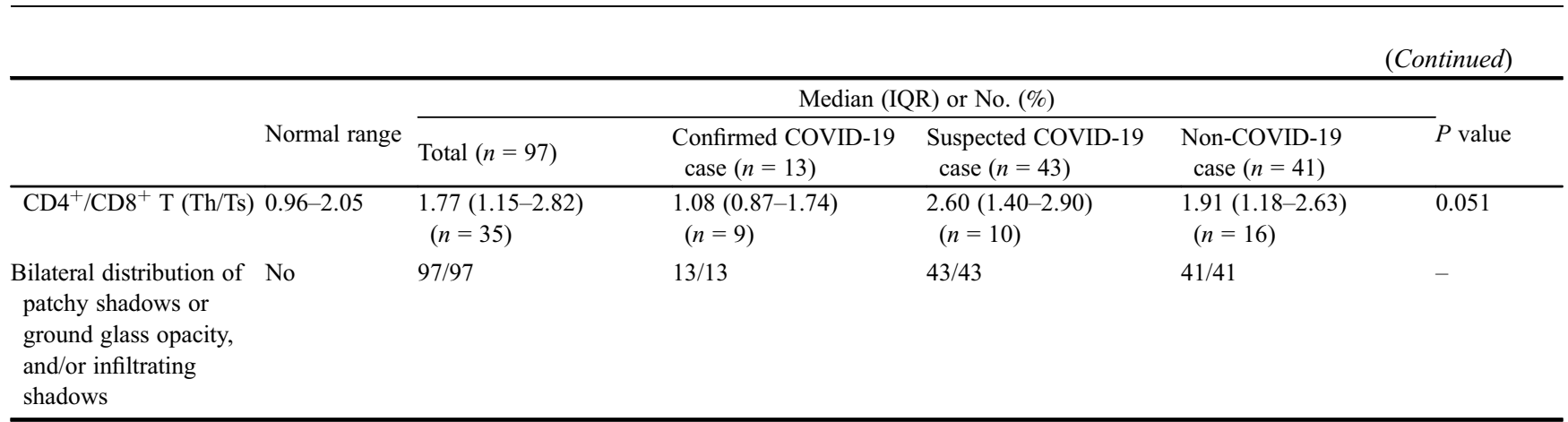

$* P<0.05$.

Abbreviations: CRP, C-reactive protein; PCT, procalcitonin; PT, prothrombin time; APTT, activated partial thromboplastin time; ALT, alanine aminotransferase; AST, aspartate aminotransferase; LDH, lactate dehydrogenase; CK, creatine kinase; BUN, blood urea nitrogen; Cr, creatinine; IL, interleukin.

and tumor necrosis factor (TNF)- $\alpha$ levels were normal. No differences were found in IL-6 and IL-10 levels among confirmed, suspected, and non-COVID-19 cases $(P>0.05)$. Out of 36 patients, counts of $\mathrm{CD}^{+} \mathrm{T}$ cells, $\mathrm{CD}^{+}{ }^{+} \mathrm{T}$ cells, and $\mathrm{CD} 16^{+} \mathrm{CD} 6^{+}$natural killer (NK) cells were below normal in $5(14.3 \%), 10(28.6 \%)$, and 13 (37.1\%) cases, respectively (Table 2). Counts of helper T cells $\left(\mathrm{CD}^{+}\right)$, suppressor $\mathrm{T}$ cells $\left(\mathrm{CD}^{+}\right)$, and NK cells $\left(\mathrm{CD} 16^{+} \mathrm{CD} 56^{+}\right)$were reduced mainly in critically ill patients. No differences were observed in the numbers of $\mathrm{CD}^{+}{ }^{+} \mathrm{T}$ cells, $\mathrm{CD}^{+} \mathrm{T}$ cells, and $\mathrm{CD} 16^{+} \mathrm{CD} 56^{+} \mathrm{NK}$ cells among confirmed, suspected, and non-COVID-19 cases $(P>0.05)$.

All 97 patients underwent RT-PCR testing for SARSCoV-2 nucleic acid upon admission. The median number of tests was 3 (range, 2-5) in patients with confirmed COVID-19, 2 (range, 1-5) in those with suspected COVID-19, and 2 (range, 1-4) in those with nonCOVID-19 causes. Out of the 97 patients, 53 (54.6\%) patients underwent serum anti-SARS-CoV-2 IgM and IgG testing starting on March 5, 2020, including 11 (84.6\%) confirmed COVID-19 cases, 22 (51.2\%) suspected COVID-19 cases, and 20 (48.8\%) non-COVID-19 cases. Out of the 11 confirmed COVID-19 cases, 5 (45.5\%) and 7 $(63.6 \%)$ were positive for IgM and IgG, respectively. Out of the 22 suspected COVID-19 cases, 1 (4.5\%) was positive for IgG but negative for IgM (Table 3, Fig. 1).

Out of the 13 confirmed COVID-19 cases, 2 cases had coinfection with Mycoplasma pneumoniae and 1 case had co-infection with rotavirus. Out of the 41 non-COVID-19 cases, the most commonly detected pathogen was $M$. pneumoniae $(27,65.9 \%)$, followed by cytomegalovirus (4, $9.8 \%)$, respiratory syncytial virus $(3,7.3 \%)$, influenza B virus $(3,7.3 \%)$, influenza A virus $(2,4.9 \%)$, Epstein-Barr virus $(2,4.9 \%)$, adenovirus $(1,2.4 \%)$, Hemophilus influenzae (2, 4.9\%), Staphylococcus (2, 4.9\%), Streptococcus pneumonia $(2,4.9 \%)$, and fungi $(1,2.4 \%)$ (Table S1).

Upon admission, chest imaging abnormalities were observed in all patients. Typical findings in chest computed tomography (CT) images were bilateral distribution of patchy shadows or ground-glass opacity, and/or infiltrating shadows in the confirmed COVID-19 cases (Fig. S1).

\section{Therapy, outcome, and follow-up}

The most commonly used antiviral therapy was interferon $\alpha-2 b$ aerosol inhalation, which was administered to 13 (100\%) confirmed COVID-19 cases, 29 (67.4\%) suspected COVID-19 cases, and 27 (65.9\%) non-COVID-19 cases. Four (30.8\%) confirmed COVID-19 cases, 2 (4.7\%) suspected COVID-19 cases, and 3 non-COVID-19 cases received intravenous ribavirin. Oseltamivir was orally administered to 3 (23.1\%) confirmed COVID-19 cases, 2 (4.7\%) suspected COVID-19 cases, and 6 (14.6\%) nonCOVID-19 cases. Three (3.5\%) non-COVID-19 cases were given oral arbidol. Azithromycin was the most commonly used antibiotic (for mycoplasma infection) in 27 (65.9\%) out of 41 non-COVID-19 cases and 2 (15.4\%) out of 13 confirmed COVID-19 cases. In 19 (19.6\%)

Table 3 Serological test for the specific IgM and IgG antibodies of SARS-CoV-2

\begin{tabular}{llllll}
\hline & Normal range & \multicolumn{4}{c}{ Median (IQR) or No. (\%) } \\
\cline { 2 - 6 } & & $\begin{array}{c}\text { Confirmed COVID-19 } \\
\text { case }(n=9)\end{array}$ & $\begin{array}{c}\text { Suspected COVID-19 } \\
\text { case }(n=19)\end{array}$ & $\begin{array}{c}\text { Non-COVID-19 } \\
\text { case }(n=14)\end{array}$ & $\begin{array}{c}P \text { value } \\
\mathrm{IgM}, \mathrm{AU} / \mathrm{mL}\end{array}$ \\
$\mathrm{IgG}, \mathrm{AU} / \mathrm{mL}$ & $0-10$ & $10.65(1.20-29.62)$ & $0.75(0.38-1.09)$ & $1.02(0.45-1.76)$ & $0.007^{* *}$ \\
\hline
\end{tabular}

Quantitative analysis of the specific IgM and IgG antibodies against SARS-CoV-2 in 9 confirmed COVID-19 cases, 19 suspected COVID-19 cases, and 14 nonCOVID-19 cases are shown in Table $3 .{ }^{* *} P<0.01$. 
patients who received oxygen, invasive mechanical ventilation was required in 2 confirmed COVID-19 cases, 1 suspected COVID-19 case, and 2 critical nonCOVID-19 cases. Two (15.4\%) confirmed COVID-19 cases, 8 (18.6\%) suspected COVID-19 cases, and 2 (4.9\%) non-COVID-19 cases received intravenous immunoglobulin. Two confirmed COVID-19 cases, 2 suspected COVID-19 cases, and 5 non-COVID-19 cases received glucocorticoid therapy. Two confirmed COVID-19 cases received plasma exchange and kidney replacement therapy.

To date, 12 (92.3\%) out of the 13 confirmed COVID-19 cases and all suspected COVID-19 cases and non-COVID19 cases have been cured and discharged. One (7.7\%) confirmed COVID-19 case had intussusceptions and multiorgan failure and eventually died [6].

At 2 weeks after discharge, all patients were followed up by telephone. Only one patient was readmitted because of diarrhea, but nucleic acid retesting by RT-PCR remained negative. The other patients were in good condition with no symptoms related to COVID-19.

\section{Discussion}

The most striking feature of this cohort was that the early symptoms in these children with probable COVID-19 were apparently nonspecific or covered by other diseases. Although we strictly adhered to two pre-examinations and triage for all outpatients, most of the probable and confirmed COVID-19 cases were admitted from the fever clinic, and $12.5 \%$ (97/773) of the probable COVID-19 cases (including 5.3\% (13/245) of confirmed COVID-19 cases) with atypical symptoms or critical illness were admitted from the emergency department. The initial symptoms of these patients did not always include fever or cough. Some pediatric patients were brought to the emergency department because of digestive tract symptoms (vomiting, diarrhea, and abdominal pain), nervous system symptoms (convulsion), or even trauma. Only when chest CT showed signs of pneumonia, they were hospitalized as probable or confirmed COVID-19 cases. Therefore, this approach was different from that of Lu et al. [6]. Owing to the parents' fear that their children were infected with SARS-CoV-2, the median time from symptom onset to hospital admission was shorter for confirmed COVID-19 cases (2.0 days) than that for suspected COVID-19 cases (3.0 days) and non-COVID19 cases $(4.0$ days $)(P<0.05)$. However, because of the mandatory isolation period, the median length of hospital stay was longer for confirmed COVID-19 cases (15.0 days) than that for suspected COVID-19 cases (10.0 days) and non-COVID-19 cases (9.0 days) $(P=0.08)$.

The clinical symptoms of COVID-19 in children differ from those in adults [10]. Most children with COVID-19 had mild symptoms or were asymptomatic. In our study, the main symptoms in confirmed COVID-19 cases during hospitalization were fever (53.5\%), diarrhea (38.5\%), vomiting (30.8\%), shortness of breath $(30.8 \%)$, cough (23.1\%), and abdominal pain (23.1\%). Compared with suspected COVID-19 and non-COVID-19 cases, the incidence of gastrointestinal symptoms (diarrhea and abdominal pain) was higher in confirmed COVID-19 cases $(P<0.05)($ Table 1$)$.

SARS-CoV-2 infection in children is mainly the result of family aggregation [11,12]. Our study showed that 10 (76.9\%) out of the 13 confirmed COVID-19 cases had documented exposure to one or more other adult family members with confirmed or suspected COVID-19. Thus, aggregative onset is an important feature in pediatric cases, and it also underscores the highly contagious nature of the virus [13]. Children with COVID-19 who are asymptomatic or have coexisting conditions can be misdiagnosed and may become potential vectors for virus transmission.

Similar to adult patients with COVID-19, most children have normal or only slightly reduced leukocyte count without evident organ dysfunction $[14,15]$. Severely ill adult patients often have coexisting medical conditions $[14,16]$. Although severe cases are less frequent among children, they can progress to death when they have coexisting conditions [6]. We analyzed cytokine (IL-2, IL4, IL-6, IL-10, and TNF- $\alpha$ ) levels and cellular immune functions $\left(\mathrm{CD}^{+}{ }^{+} \mathrm{T}\right.$ cells, $\mathrm{CD} 4^{+} \mathrm{T}$ cells, $\mathrm{CD}^{+} \mathrm{T}$ cells, and $\mathrm{CD} 16^{+} \mathrm{CD} 56^{+} \mathrm{NK}$ cells) in some patients and found that the increase in IL- 6 and IL-10 levels and the decrease in numbers of helper $\mathrm{T}$ cells $\left(\mathrm{CD} 4^{+}\right)$, suppressor $\mathrm{T}$ cells $\left(\mathrm{CD} 8^{+}\right)$, and NK cells $\left(\mathrm{CD} 16^{+} \mathrm{CD} 56^{+}\right)$mainly occurred in severe cases with no difference between confirmed, suspected, and non-COVID-19 cases $(P>0.05)$ (Table 2). This result suggested that the virus causes direct damage to organs and that further impairment of organ function related to cytokine storm induced by the viral invasion in critical cases is not unique to SARS-CoV-2 infection because it was also found in children that were severely infected with other pathogens.

As with SARS and MERS, children with SARS-CoV-2 infection have low morbidity [17-21]. The reason for this phenomenon is unknown, but it has been attributed to the fact that children have a more active innate immune response, healthier respiratory tracts, and fewer underlying disorders than adults. A more vigorous immune response in adults may also explain the detrimental immune response in acute respiratory distress syndrome [22]. SARS-CoV-2, a newly emerged virus, binds with high affinity to human angiotensin converting enzyme 2 (ACE2) and employs it as an entry receptor to invade target cells [23]. The ACE2 protein is abundantly expressed in lung alveolar epithelial cells and enterocytes of the small intestines and is present in arterial and venous endothelial cells and arterial smooth muscle cells of all 
organs [24,25]. Therefore, SARS-CoV-2 may infect patients not only through the respiratory tract in the form of air droplets but also through the digestive tract by contact or fecal-oral transmission.

We previously described chest imaging features in five COVID-19 cases with nonrespiratory symptoms as the first manifestation in children [26]. In the early phase of the disease, chest X-rays showed no abnormalities, potentially leading to missed diagnosis. As in adults, chest CT images in children with COCID-19 revealed the presence of viral pneumonia, with early lesions frequently detected in the lung periphery and showing ground-glass opacity and/or infiltrating shadows. With disease progression, the lesions increased in number and expanded to involve multiple lung lobes. In the serious cases, the CT images showed diffuse consolidation in the lungs, as well as so-called white lung and air bronchogram.

RT-PCR was one of the first laboratory diagnostic methods to be established in this pandemic and can yield results within $2 \mathrm{~h}$. In our study, 13 out of the 97 children were confirmed to be positive for SARS-CoV-2 infection by this method. Owing to the sudden outbreak of COVID19 , detection kits varying in sensitivity and specificity were used for testing, and cases that were highly suspected of SARS-CoV-2 infection were sometimes found to be negative although they could not be completely ruled out [27]. In fact, nucleic acid testing of nasal and pharyngeal swabs has a detection time window [28]. Dynamic monitoring of SARS-CoV-2 nucleic acid profiles by RTPCR showed that the positive rate of oral swabs gradually decreases, whereas that of anal swabs increases with the duration of hospitalization [12]. At the same time, serum IgM positivity increased from 50\% (8/16) upon admission to $81 \%$ (13/16) after 5 days of hospitalization, whereas IgG positivity rate increased from $81 \%(13 / 16)$ to $100 \%(16 /$ 16) [29]. Therefore, to improve the reliability of diagnosis and avoid false-negative results, different biological specimens (nasal and pharyngeal swabs, anal swab, and blood) should be used for nucleic acid testing, and serum $\operatorname{IgM}$ and IgG levels should be monitored. Serological tests can serve as an important supplementary tool for COVID19 diagnosis. Therefore, serologic testing must be conducted in children who are highly suspected of having COVID-19 but who have tested negative via RT-PCR.

Notably, in the 97 children with probable COVID-19 who were initially admitted from the emergency department, the most frequently detected pathogen was $M$. pneumonia in 27 (65.9\%) out of the 41 non-COVID cases and $2(15.4 \%)$ out of the 13 confirmed COVID-19 cases. Additionally, out of the 41 non-COVID-19 cases, we detected other viral infections in 15 cases, bacterial infections in 6 cases, and fungal infection in 1 case. Four cases had coinfection with two pathogens, and two cases had coinfection with three pathogens. Therefore, clinical manifestations, laboratory test results, and findings from chest imaging of children with COVID-19 can be nonspecific, and these cases cannot be definitively diagnosed as COVID-19 unless etiological analysis is performed. In particular, coinfection with another pathogen - especially $M$. pneumonia - could mask the symptoms of the primary infection.

Our study had some limitations. First, it was a retrospective, single-center study with a small sample of patients admitted by the emergency department. Standardized data from a larger prospective cohort study could provide more information on the features of COVID-19 in children. Second, we only conducted serologic testing in a subset of our patients and found just one case that was positive for SARS-CoV-2 specific IgG in suspected COVID-19 cases. Detection of nucleic acid in different biological specimens of suspected COVID-19 cases and dynamic monitoring of serum antibodies could reveal additional cases and thus reduce the risk of missed diagnosis. Finally, according to the standards of Experts' Consensus Statement [7], suspected cases may be considered as confirmed based on supportive evidence from nucleic acid or serologic testing. However, serologic testing was not initiated until March 5, 2020 and was only performed in some children ( 25 cases). To ensure the consistent application of diagnostic criteria to all patients, we used nucleic acid test results as the basis for diagnosis, and this approach may have resulted in some cases being overlooked. To address this potential omission, we are currently following up these children and advising them to undergo serologic testing.

In summary, our findings highlighted the characteristics of COVID-19 in pediatric patients admitted from the emergency department, which differ from those admitted from the fever clinic [6]. In children with similar clinical manifestations and even chest $\mathrm{CT}$ images, distinguishing COVID-19 from pneumonia caused by other pathogens (e.g., M. pneumoniae) before etiologic analysis (nucleic acid and serological testing) is impossible. Moreover, serologic testing can serve as an important adjunctive method for COVID-19 diagnosis, especially when the patient is highly suspected of SARS-CoV-2 infection but is found to be negative by nucleic acid testing.

\section{Acknowledgements}

We acknowledge all healthcare workers involved in the diagnosis and treatment of patients in Wuhan Children's Hospital. This study was supported by the National Natural Science Foundation of China (No. 81741099).

\section{Compliance with ethics guidelines}

Xiaofang Cai, Hanlan Jiang, Simin Zhang, Shengying Xia, Wenhui $\mathrm{Du}$, Yaoling Ma, Tao Yu, and Wenbin Li declare that they have no conflicts of interest. All procedures followed were in accordance 
with the ethical standards of the responsible committee on human experimentation (institutional and national) and with the Helsinki Declaration of 1975, as revised in 2000 (5). Oral consent was obtained from the legal guardians in this study.

Electronic Supplementary Material Supplementary material is available in the online version of this article at https://doi.org/ $10.1007 / \mathrm{s} 11684-020-0820-7$ and is accessible for authorized users.

\section{References}

1. Zhou M, Zhang X, Qu J. Coronavirus disease 2019 (COVID-19): a clinical update. Front Med 2020; 14(2): 126-135

2. Xu J, Chen Y, Chen H, Cao B. 2019 novel coronavirus outbreak: a quiz or final exam? Front Med 2020; 14(2): 225-228

3. Wang Y, Wang Y, Chen Y, Qin Q. Unique epidemiological and clinical features of the emerging 2019 novel coronavirus pneumonia (COVID-19) implicate special control measures. J Med Virol 2020: 92(6):568-576

4. Lai CC, Shih TP, Ko WC, Tang HJ, Hsueh PR. Severe acute respiratory syndrome coronavirus 2 (SARS-CoV-2) and coronavirus disease-2019 (COVID-19): the epidemic and the challenges. Int J Antimicrob Agents 2020; 55(3): 105924

5. Zhang Z, Liu S, Xiang M, Li S, Zhao D, Huang C, Chen S. Protecting healthcare personnel from 2019-nCoV infection risks: lessons and suggestions. Front Med 2020; 14(2): 229-231

6. Lu X, Zhang L, Du H, Zhang J, Li YY, Qu J, Zhang W, Wang Y, Bao S, Li Y, Wu C, Liu H, Liu D, Shao J, Peng X, Yang Y, Liu Z, Xiang Y, Zhang F, Silva RM, Pinkerton KE, Shen K, Xiao H, Xu S, Wong GWK; Chinese Pediatric Novel Coronavirus Study Team. SARS-CoV-2 infection in children. N Engl J Med 2020; 382(17): $1663-1665$

7. Shen K, Yang Y, Wang T, Zhao D, Jiang Y, Jin R, Zheng Y, Xu B, Xie Z, Lin L, Shang Y, Lu X, Shu S, Bai Y, Deng J, Lu M, Ye L, Wang X, Wang Y, Gao L, China National Clinical Research Center for Respiratory Diseases; National Center for Children's Health, Beijing, China; Group of Respirology, Chinese Pediatric Society, Chinese Medical Association; Chinese Medical Doctor Association Committee on Respirology Pediatrics; China Medicine Education Association Committee on Pediatrics; Chinese Research Hospital Association Committee on Pediatrics; Chinese Non-government Medical Institutions Association Committee on Pediatrics; China Association of Traditional Chinese Medicine, Committee on Children's Health and Medicine Research; China News of Drug Information Association, Committee on Children's Safety Medication; Global Pediatric Pulmonology Alliance. Diagnosis, treatment and prevention of 2019 novel coronavirus infection in children: experts' consensus statement. World J Pediatr 2020; 16(3): 223-231

8. Holshue ML, DeBolt C, Lindquist S, Lofy KH, Wiesman J, Bruce H, Spitters C, Ericson K, Wilkerson S, Tural A, Diaz G, Cohn A, Fox L, Patel A, Gerber SI, Kim L, Tong S, Lu X, Lindstrom S, Pallansch MA, Weldon WC, Biggs HM, Uyeki TM, Pillai SK, Washington State 2019-nCoV Case Investigation Team. First case of 2019 novel coronavirus in the United States. N Engl J Med 2020; 382(10): 929-936
9. Society of Pediatrics, Chinese Medical Association; Editorial Board, Chinese Journal of Pediatrics. Recommendations for the diagnosis, prevention and control of the 2019 novel coronavirus infection on children (first interim edition). Chin J Pediatr (Zhonghua Er Ke Za Zhi) 2020; 58(3): 169-174 (in Chinese)

10. Li LQ, Huang T, Wang YQ, Wang ZP, Liang Y, Huang TB, Zhang HY, Sun WM, Wang YP. COVID-19 patients' clinical characteristics, discharge rate, and fatality rate of meta-analysis. J Med Virol 2020; 92(6): 577-583

11. Wei M, Yuan J, Liu Y, Fu T, Yu X, Zhang ZJ. Novel coronavirus infection in hospitalized infants under 1 year of age in China. JAMA 2020; 323(13): 1313-1314

12. Zhang YH, Lin DJ, Xiao MF, Wang JC, Wei Y, Lei ZX, Zeng ZQ, Li L, Li HA, Xiang W. 2019-novel coronavirus infection in a threemonth-old baby. Chin J Pediatr (Zhonghua Er Ke Za Zhi) 2020; 58 (3): 182-184 (in Chinese)

13. Cao Q, Chen YC, Chen CL, Chiu CH. SARS-CoV-2 infection in children: transmission dynamics and clinical characteristics. J Formos Med Assoc 2020; 119(3): 670-673

14. Wang D, Hu B, Hu C, Zhu F, Liu X, Zhang J, Wang B, Xiang H, Cheng Z, Xiong Y, Zhao Y, Li Y, Wang X, Peng Z. Clinical characteristics of 138 hospitalized patients with 2019 novel coronavirus-infected pneumonia in Wuhan, China. JAMA 2020; 323(11): 1061-1069

15. Qin C, Zhou L, Hu Z, Zhang S, Yang S, Tao Y, Xie C, Ma K, Shang K, Wang W, Tian DS. Dysregulation of immune response in patients with COVID-19 in Wuhan, China. Clin Infect Dis 2020; 71(15): 762-768

16. Huang C, Wang Y, Li X, Ren L, Zhao J, Hu Y, Zhang L, Fan G, Xu J, Gu X, Cheng Z, Yu T, Xia J, Wei Y, Wu W, Xie X, Yin W, Li H, Liu M, Xiao Y, Gao H, Guo L, Xie J, Wang G, Jiang R, Gao Z, Jin Q, Wang J, Cao B. Clinical features of patients infected with 2019 novel coronavirus in Wuhan, China. Lancet 2020; 395(10223): 497506

17. Zeng LK, Tao XW, Yuan WH, Wang J, Liu X, Liu ZS. First case of neonate infected with novel coronavirus pneumonia in China. Chin J Pediatr (Zhonghua Er Ke Za Zhi) 2020; [Epub ahead of print] (in Chinese)

18. Chen F, Liu ZS, Zhang FR, Xiong RH, Chen Y, Cheng XF, Wang WY, Ren J. First case of severe childhood novel coronavirus pneumonia in China. Chin J Pediatr (Zhonghua Er Ke Za Zhi) 2020; 58(3): 179-183 (in Chinese)

19. Memish ZA, Al-Tawfiq JA, Assiri A, AlRabiah FA, Al Hajjar S, Albarrak A, Flemban H, Alhakeem RF, Makhdoom HQ, Alsubaie S, Al-Rabeeah AA. Middle East respiratory syndrome coronavirus disease in children. Pediatr Infect Dis J 2014; 33(9): 904-906

20. Bitnun A, Allen U, Heurter H, King SM, Opavsky MA, Ford-Jones EL, Matlow A, Kitai I, Tellier R, Richardson S, Manson D, Babyn P, Read S, Other Members of the Hospital for Sick Children SARS Investigation Team. Children hospitalized with severe acute respiratory syndrome-related illness in Toronto. Pediatrics 2003; 112(4): e261

21. Wu Z, McGoogan JM. Characteristics of and important lessons from the coronavirus disease 2019 (COVID-19) outbreak in China: summary of a report of 72314 cases from the Chinese Center for Disease Control and Prevention. JAMA 2020; 323(13): 1239

22. Lee PI, Hu YL, Chen PY, Huang YC, Hsueh PR. Are children less 
susceptible to COVID-19? J Microbiol Immunol Infect 2020; 53(3): 371-372

23. Walls AC, Park YJ, Tortorici MA, Wall A, McGuire AT, Veesler D. Structure, function, and antigenicity of the SARS-CoV-2 spike glycoprotein. Cell 2020; 181(2): 281-292.e6

24. Hamming I, Timens W, Bulthuis ML, Lely AT, Navis G, van Goor H. Tissue distribution of ACE2 protein, the functional receptor for SARS coronavirus. A first step in understanding SARS pathogenesis. J Pathol 2004; 203(2): 631-637

25. Jia H. Pulmonary angiotensin-converting enzyme 2 (ACE2) and inflammatory lung disease. Shock 2016; 46(3): 239-248

26. Cai X, Ma Y, Li S, Chen Y, Rong Z, Li W. Clinical characteristics of 5 COVID-19 cases with non-respiratory symptoms as the first manifestation in children. Front Pediatr 2020; 8: 258

27. Chan JF, Yip CC, To KK, Tang TH, Wong SC, Leung KH, Fung
AY, Ng AC, Zou Z, Tsoi HW, Choi GK, Tam AR, Cheng VC, Chan $\mathrm{KH}$, Tsang OT, Yuen KY. Improved molecular diagnosis of COVID-19 by the novel, highly sensitive and specific COVID-19$\mathrm{RdRp} / \mathrm{Hel}$ real-time reverse transcription-polymerase chain reaction assay validated in vitro and with clinical specimens. J Clin Microbiol 2020; 58(5): e00310-20

28. Liu R, Han H, Liu F, Lv Z, Wu K, Liu Y, Feng Y, Zhu C. Positive rate of RT-PCR detection of SARS-CoV-2 infection in 4880 cases from one hospital in Wuhan, China, from Jan to Feb 2020. Clin Chim Acta 2020; 505: 172-175

29. Zhang W, Du RH, Li B, Zheng XS, Yang XL, Hu B, Wang YY, Xiao GF, Yan B, Shi ZL, Zhou P. Molecular and serological investigation of 2019-nCoV infected patients: implication of multiple shedding routes. Emerg Microbes Infect 2020; 9(1): 386389 